Bull. Mater. Sci., Vol. 14, No. 6, December 1991, pp. 1331-1341. (C) Printed in India.

\title{
Grain boundary effect on the electrical properties of boron-doped polysilicon films
}

\author{
N K UPRETI and S SINGH \\ Department of Physics, G. B. Pant University of Agriculture and Technology, Pantragar \\ 263145 , India \\ MS received 22 October 1990; revised 13 February 1991

\begin{abstract}
The effect of grain boundary width has been accounted for and a modified simple model of average carrier concentration is presented considering the transport mechanism of charge carriers by thermionic emission only. It is found that the electrical properties of polysilicon are very sensitive to doping concentration when the grain size is small and the effect of grain boundary width on electrical properties increases as the grain size decreases. The inclusion of grain boundary width in resistivity and mobility formulae also gives better results near the critical doping concentration. The proposed model gives better agreement between experimental data and theoretical results.
\end{abstract}

Keywords. Grain boundary; electrical properties; polysilicon films; thermionic emission.

\section{Introduction}

No material as yet seems to be superior to single crystal silicon in converting solar energy into other useful forms of energy. Solar cells using monocrystal silicon as a material for photovoltaic conversion are reasonably efficient, but are expensive to fabricate. Therefore, polysilicon has become one of the most promising materials for the realization of low-cost solar cells for terrestrial as well as other applications.

The present work explains the electrical properties of boron-doped polycrystalline silicon films in dark considering carrier transport by thermionic emission only and treated through Fermi statistics. Several earlier workers (Kamins 1971; Cowher and Sedwich 1972; Choudhary and Hower 1973; Fripp and Slack 1973; Seto 1975; Lu et al 1981a) while presenting their models neglected the effect of grain boundary considering its marginal width. In order to better explain the experimental results we found that the inclusion of finite width of grain boundary was very important to get better results particularly near the critical doping range. We present a simple and modified model of average carrier concentration.

\section{Theory}

To explain the electrical properties of boron-doped polysilicon, we assume that the grain boundary trapping states of density $N_{\mathrm{gs}} \mathrm{cm}^{-2}$ are located in narrow energy range at energy $E_{T}$ (referred to as intrinsic Fermi level $E_{i 0}$ at the centre of the grain), shown in figure 1 ( $\mathrm{Lu}$ et al 1980). Although polysilicon is a three-dimensional substance with grains having a distribution of sizes and irregular shapes, in order to simplify the theory we assume that the polysilicon was composed of identical cubic grains of average size $d$ and its transport properties to be one-dimensional. Also $N_{\mathrm{gs}}$ and $E_{T}$ were independent of doping concentration ( $\mathrm{Lu}$ et al 1981b). 


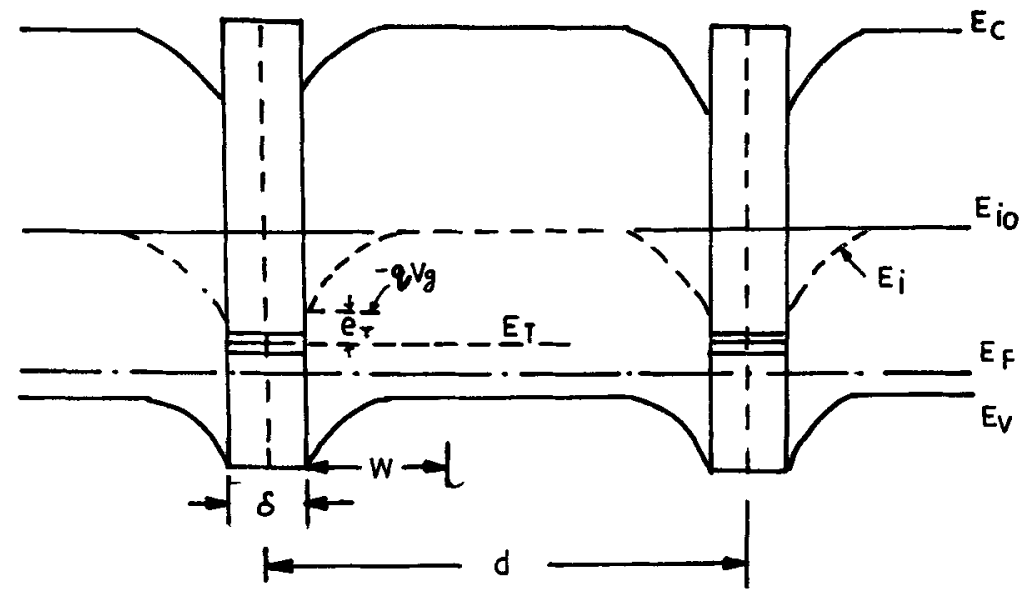

Figure 1. Energy band diagram of p-type silicon.

When polysilicon film was implanted with one type of impurity most of the dopants entered the crystallite lattice substitutionally and were assumed to be uniformly distributed throughout the film after subsequent thermal treatment. An impurity level was formed inside the crystallite, and the impurity atoms were ionized to create majority mobile carriers. The traps in the grain boundary charged by trapping mobile carriers depleted the regions in the crystallite, and the potential barriers $\left(V_{g}\right)$ were thus formed on both sides of the grain boundary. The electrical properties of polycrystalline silicon were governed by these potential barriers. This potential barrier is given as

$$
V(x)=V_{g} \quad 0 \leq / x / \leq \delta / 2,
$$

where $\delta$ stands for grain-boundary width. This space-charge potential barrier was given by (Lu et al 1981b)

$$
V_{g}= \pm\left(q N W^{2} / 2 \varepsilon\right)
$$

where $\varepsilon$ denotes the dielectric permittivity of polysilicon, plus denotes $p$-type dopants and minus indicates $n$-type, and $W$ the depletion region width.

For p-type dopants, Fermi level $E_{F}$ can be calculated using the charge neutrality condition, i.e. equating the net negative charge density in the depletion region to the net positive charge density trapped at trapping states i.e. ( $\mathrm{Lu}$ et al 1981b)

$$
2 N W=N_{\mathrm{gs}}^{+}=\frac{N_{\mathrm{gs}}}{2 \exp \left[\left(E_{\mathrm{F}}-e_{T}+q V_{g}\right) / K T\right]+1} \text {. }
$$

Here $N_{\mathrm{gs}}^{+}$is the ionized effective trap-density in the grain boundary, $e_{T}$ the trapping state energy referred to as $E_{i}$ at grain boundary. $E_{i}$ is the intrinsic Fermi level referred to as $E_{i 0}$, the intrinsic Fermi level at the centre of the grain.

We now know that $N_{\mathrm{gs}}^{+}$and grain size $d$ are finite quantities. Hence there exists a certain doping concentration $N^{*}$, known as critical doping concentration, such that if doping concentration $N<N^{*}$, the grains are assumed to be completely depleted and in this case the depletion region width is given as:

$$
2 W=a-\delta \text {. }
$$


On the other hand, if $N>N^{*}$ the grains are partially depleted and in this case $2 W<d-\delta$. Both cases are separately discussed as follows: (a) Completely depleted region: In this case $N<N^{*}$ and $W$ are given by (4). Considering only for $p$-type dopants and putting (4) into (2), one gets

$$
V_{g}=\left[q N(d-\delta)^{2}\right] / 8 \varepsilon .
$$

From (3) the Fermi level is given as

$$
E_{F}=e_{T}-q V_{g}+K T \ln \left[\frac{1}{2}\left(\frac{N_{g s}}{N(d-\delta)}-1\right)\right] .
$$

(b) partially depleted region: In this case $N>N^{*}$ and $2 W<d-\delta$. Naturally in this case the crystal has both depletion and neutral regions. In neutral region the ionized impurity concentration $N^{+}$at temperature $T$ (Sze 1981) is :

$$
N^{+}=\frac{N}{1+2 \exp \left[\left(E_{A}-E_{F}\right) / K T\right]},
$$

where $E_{A}$ is the acceptor impurity level within the forbidden band gap $E_{g}$, and for boron it is given as ( $\mathrm{Lu}$ et al 1981b)

$$
E_{A}=-\frac{1}{2} E_{g}+0.08-4.3 \times 10^{-8} N^{1 / 3}
$$

Here $E_{g}$ is band-gap for silicon in $\mathrm{eV}$. This hole concentration in the neutral region is expressed as (Sze 1981):

$$
P=N^{+}=n_{i} \exp \left(-E_{F} / K T\right),
$$

where $n_{i}$ is the intrinsic carrier concentration. Combining (7) and (9) one gets

$$
n_{i} \exp \left(-E_{F} / K T\right)=\frac{N}{1+2 \exp \left[\left(E_{A}-E_{F}\right) / K T\right]} .
$$

Solving this equation we get the Fermi level for the partially depleted region as

$$
E_{F}=-K T \ln \frac{-n_{i}+\left[n_{l}^{2}+8 n_{i} N \exp \left(E_{A} / K T\right)\right]^{1 / 2}}{4 n_{i} \exp \left(E_{A} / K T\right)}
$$

\subsection{Average carrier concentration}

We know that in the case of partially-depleted grains, the grain has both depletion and neutral regions. The density of mobile holes in the undepleted part of the crystallite is

$$
P_{g}=P(d-\delta-2 W) .
$$

Now, if $V_{g} / W$ is the average electric field near the grain boundary region, then the density of free carriers at any distance $x$ from the grain boundary within the depleted region can be expressed as (Henry et al 1978)

$$
P(x) \approx P \exp \frac{q V_{g}}{K T}(x / w-1) .
$$

Using the above equation we can calculate the total density of free carriers in the 
depleted region of the crystallite as

$$
\begin{aligned}
P_{b} & =2 P \int_{0}^{W} \exp \frac{q V_{g}}{K T}(x / w-1) \mathrm{d} x \\
\text { or } \quad P_{b} & =\frac{2 P K T W}{q V_{g}}\left[1-\exp \left(-q V_{g} / K T\right)\right] .
\end{aligned}
$$

The average carrier concentration $P^{*}$ in the grain is defined as

$$
P^{*}=\left(P_{g}+P_{b}\right) / d
$$

Using (12), (14) and (15) the average carrier concentration in a partially depleted grain is given as:

$$
P^{*}=n_{i} \exp \left(-E_{F} / K T\right)\left[1-\frac{\delta}{d}-\frac{2 W}{d}\left\{1-\frac{K T}{q V_{g}}+\frac{K T}{q V_{g}} \exp \left(\frac{-q V_{g}}{K T}\right)\right\}\right]
$$

Putting in this equation $d-\delta=2 W$, the average carrier concentration in a completely depleted grain is given as

$$
\begin{aligned}
& P^{*}=2 W n_{i} / d \exp \left(-E_{F} / K T\right)\left[K T / q V_{g}-K T / q V_{g} \exp \left(-q V_{g} / K T\right)\right], \\
& \text { or } \quad P^{*}=2 W K T n_{i} / d q V_{g} \exp \left(-E_{F} / K T\right)\left[1-\exp \left(-q V_{g} / K T\right)\right] .
\end{aligned}
$$

\subsection{Resistivity and mobility}

In polysilicon the total resistivity is mainly composed of barrier resistivity and crystallite bulk resistivity. The total resistivity $\rho$ which includes $\rho_{b}$ and $\rho_{c}$ after proper simplification is given as (Upreti and Singh 1987)

$$
\rho=1 / f d q^{2} p\left(2 \pi m_{h}^{*} K T\right)^{\frac{1}{2}} \exp \left(q V_{g} / K T\right)+\rho_{c}\left(1-\frac{2 W+\delta}{d}\right) .
$$

Here $f$ is the scaling factor. The bulk resistivity of the crystallite neutral region was equal to the resistivity of the single crystal silicon (Wolf 1969). Hence in the above equation, $\rho_{c}$ is taken as monocrystalline silicon resistivity (Muller and Kamins 1977). The effective mobility for polysilicon can therefore be given as

$$
\mu_{\mathrm{eff}}=1 / P^{*} \rho q
$$

where $P^{*}$ and $\rho$ are given by (16) and (17) respectively.

\section{Results and discussion}

Equation (16) shows that average carrier concentration in polysilicon films is mainly determined by the doping concentration, grain size and temperature. The variation of average carrier concentration $\left(P^{*}\right)$ with doping concentration $(N)$ for different values of grain size $(d)$ is shown in figure 2 . For calculations, use has been made of parameters listed in tables 1 and 2. Table 3 demonstrates the superiority of our model over that of Lu et al (1981b). For $N<N^{*}$ the grain is completely 


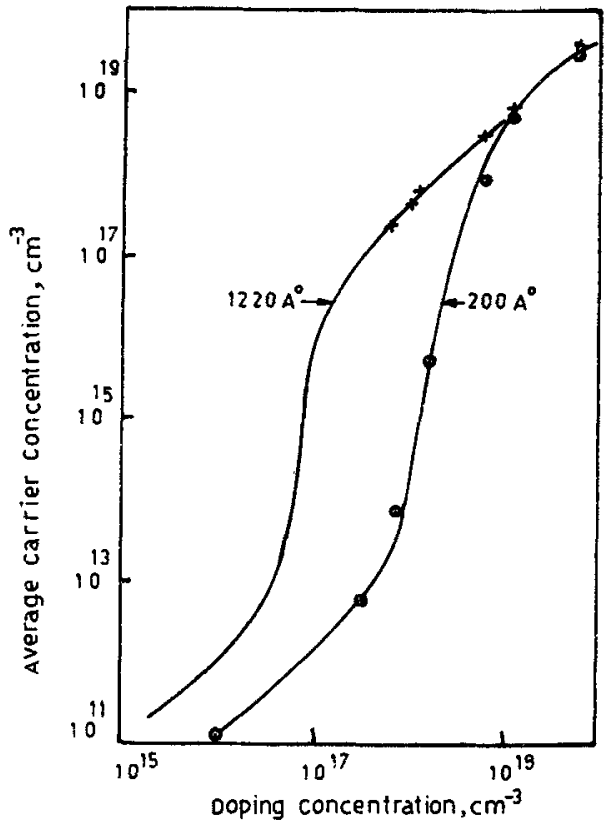

Figure 2. Theoretical variation of average carrier concentration with doping concentrafion at room temperature for polysilicon with different grain sizes: + , data are from Lu et al (1981b); $\odot$, from Seto (1975); - , present work.

Table 1. Parameter values used in calculations with various grain sizes

\begin{tabular}{lccccc}
\hline $\begin{array}{l}\text { Experimental } \\
\text { data source }\end{array}$ & $\begin{array}{c}e_{T} \\
(\mathrm{eV})\end{array}$ & $\begin{array}{c}N_{\mathrm{gs}} \\
\left(\mathrm{cm}^{-2}\right)\end{array}$ & $\begin{array}{c}d \\
(\AA)\end{array}$ & $f$ & $\delta(\AA)$ \\
\hline Seto $(1975)$ & -0.18 & $3.34 \times 10^{12}$ & 200 & 0.12 & 8.5 \\
Lu et al $(1981 \mathrm{~b})$ & -0.17 & $1.9 \times 10^{12}$ & 1220 & 0.06 & 8.5 \\
\hline
\end{tabular}

Table 2. Numerical values of parameters used in calculations (Wolf 1969)

\begin{tabular}{ll}
\hline Parameters & Value \\
\hline$E_{g}$ & $1.12 \mathrm{eV}$ \\
$n_{i}$ & $1.45 \times 10^{10} \mathrm{~cm}^{-3}$ \\
$\varepsilon$ & $11.7 \varepsilon_{0}$ \\
$m_{h}^{*}$ & $0.386 m_{0}$ \\
\hline
\end{tabular}

depleted off the free carriers and $P^{*} \ll N$, because most of the carriers are trapped by the grain boundary states. As the doping concentration increases, the traps are filled and $P^{*}$ approaches near $N$. For $N \gg N^{*}$, most of the traps are filled and consequently the space-charge barriers will be very narrow. Thus at high doping levels $P^{*} \approx N$. For $N>N^{*}$, the localized states of boron broaden into a band, which in turn, merges with the nearby band continuum. Instead of discrete energy levels, a new set of energy bands appears and the Fermi level at $T=0$ lies inside one of these bands (Pantalides et al 1985). This lends support to our arguments. 
Table 3. Room temperature average carrier concentration with different doping concentrations for $d=1220 \AA$ and $\delta=8.5 \AA$

\begin{tabular}{lccc}
\hline $\begin{array}{l}\text { Doping concentra- } \\
\text { tion }\left(\mathrm{cm}^{-3}\right)\end{array}$ & $\begin{array}{c}\text { Measured } P^{*} \\
\left(\mathrm{~cm}^{-3}\right)\end{array}$ & $\begin{array}{c}\text { Calculated } \\
\left(\mathrm{cm}^{-3}\right)\end{array}$ & $\begin{array}{c}\text { Calculated } \\
\left(\mathrm{cm}^{-3}\right)\end{array}$ \\
\hline $5 \times 10^{17}$ & $3.0 \times 10^{17}$ & $3.6 \times 10^{17}$ & $3.2 \times 10^{17}$ \\
$8 \times 10^{17}$ & $5.1 \times 10^{17}$ & $6.1 \times 10^{17}$ & $5.7 \times 10^{17}$ \\
$1 \times 10^{18}$ & $7.1 \times 10^{17}$ & $7.8 \times 10^{17}$ & $7.4 \times 10^{17}$ \\
$5 \times 10^{18}$ & $4.1 \times 10^{18}$ & $3.9 \times 10^{18}$ & $3.9 \times 10^{18}$ \\
\hline
\end{tabular}

${ }^{a}$ Lu et al (1981b); ${ }^{b}$ Present work

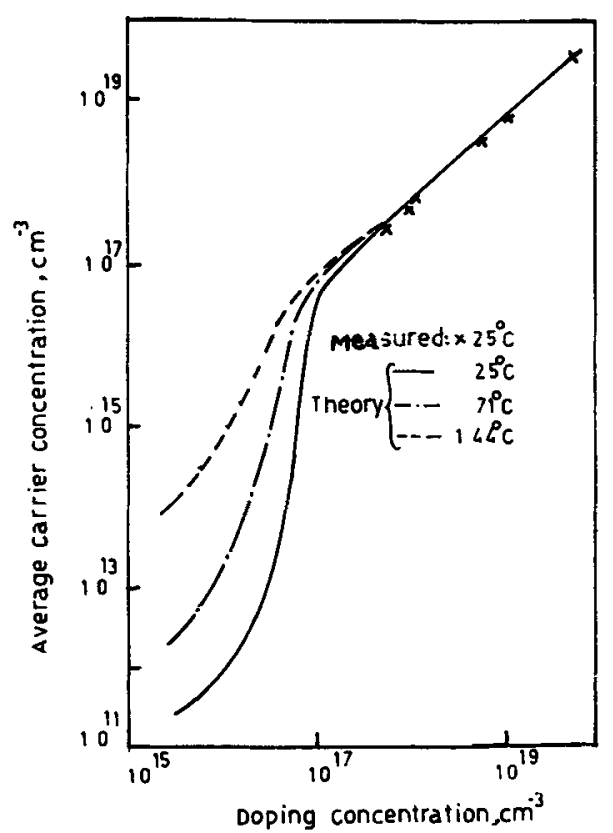

Figure 3. Theoretical variation of average carrier concentration with doping concentration at different temperatures for polysilicon with grain size $d=1220 \AA$ : + , data are from Lu et al (1981b); -, present work.

Figure 3 is a plot of average carrier concentration $P^{*}$ vs doping concentration at different temperatures. The reduction of carriers in lightly and medium-doped ranges is mostly the result of traps, but in the highly-doped regions the partial ionization of dopants caused by the existence of an impurity level becomes more important. This effect becomes significant at lower temperatures and in addition to segregation, it contributes to the smaller carrier concentration than doping concentration in highly doped samples. Figures 2 and 3 seem to be identical to that of Lu et al (1981b) but a careful glance reveals that the experimental and theoretical agreement is much better in our case. Assuming $e_{T}$ and $N_{\mathrm{gs}}$ independent of grain size, the variation of $P^{*}$ with grain size for different values of doping concentration is plotted in figure 4 . This shows that $P^{*}$ increases with increasing grain size. Also, it is clear from this figure that the effect of grain size on $P^{*}$ is large at low doping concentrations because most of the carriers are trapped. As the doping concentration increases, the effect of grain size on $P^{*}$ decreases. We think this is a 


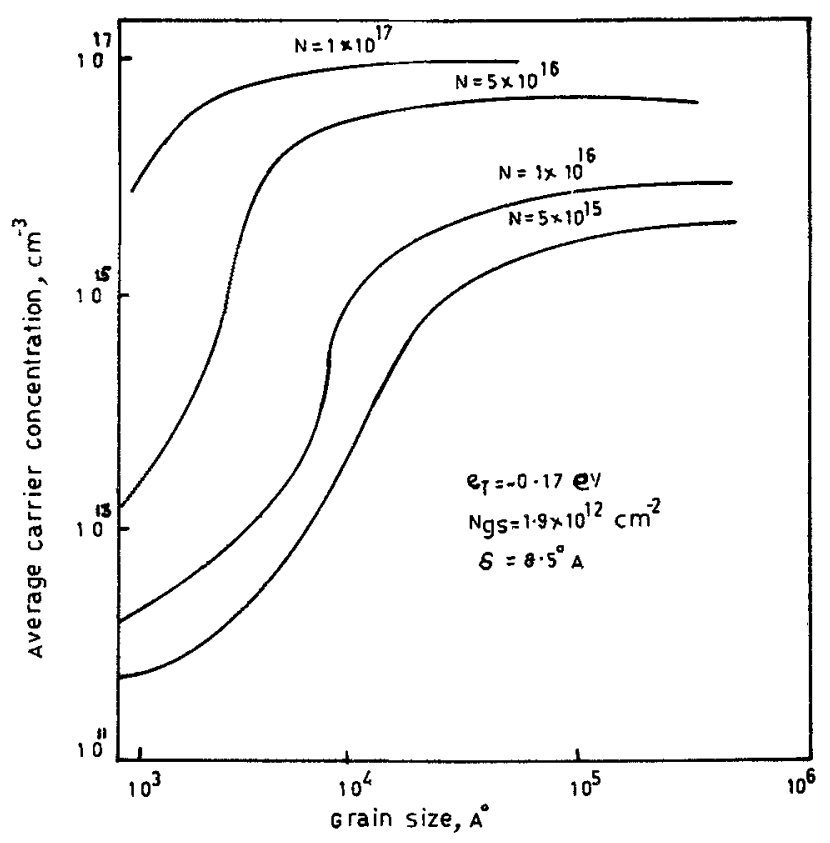

Figure 4. Computed variation of average carrier concentration in polysilicon with grain size for different values of doping concentration.

natural consequence and needs no explanation. For small grain sizes the average carrier concentration is much less than for doping concentration $\left(P^{*} \ll N\right)$. On the other hand, for large grain sizes $P^{*} \approx N$. This is an important feature as the traps are filled. Further, it is clear that the depleted grain is very sensitive to grain size. As the grain boundary width is estimated to be between $6 \AA$ and $110 \AA$ (Mandurah et al 1981; Murota and Sawai 1982) its effect on the electrical properties of polysilicon is important, particularly when the doping concentration is very high and the grain size is smaller than $400 \AA$. As the grain size increases, the effect of grain boundary width on $P^{*}$ cannot be neglected since $2 W \ll d$ as confirmed experimentally (Murota and Sawai 1982).

Figure 5 deals with a change in the potential barrier height as a function of $N$. It reveals that an increasing value of $N$ results in a corresponding linear increase of potential reaching a maximum at $N=N^{*}$, and then decreasing rapidly. It is also clear that for $d=1220 \AA$, one gets $N^{*} \approx 7 \times 10^{16} \mathrm{~cm}^{-3}$ and for $d=200 \AA$, $N^{*} \approx 1 \times 10^{18} \mathrm{~cm}^{-3}$. It is understandable in terms of non-additive interaction potentials for heavily-doped materials. By comparing equilibrium barrier heights in $p$-type polysilicon with that of Seto (1975), Samaj (1987) assumed constant distribution of interface states and showed the tendency of the barrier to decrease to the value so that the settlement of concentration of electrons and holes at the interface is facilitated for small densities of donors and high densities of interface states. This may well be true in some situations and will very much depend on the preparative parameters. This assumption of constant distribution of interface states is perhaps to be looked into further. However, $p$-type doping efficiency does depend on preparation condition and has been supported by Abo-Namous (1987) in his work. 


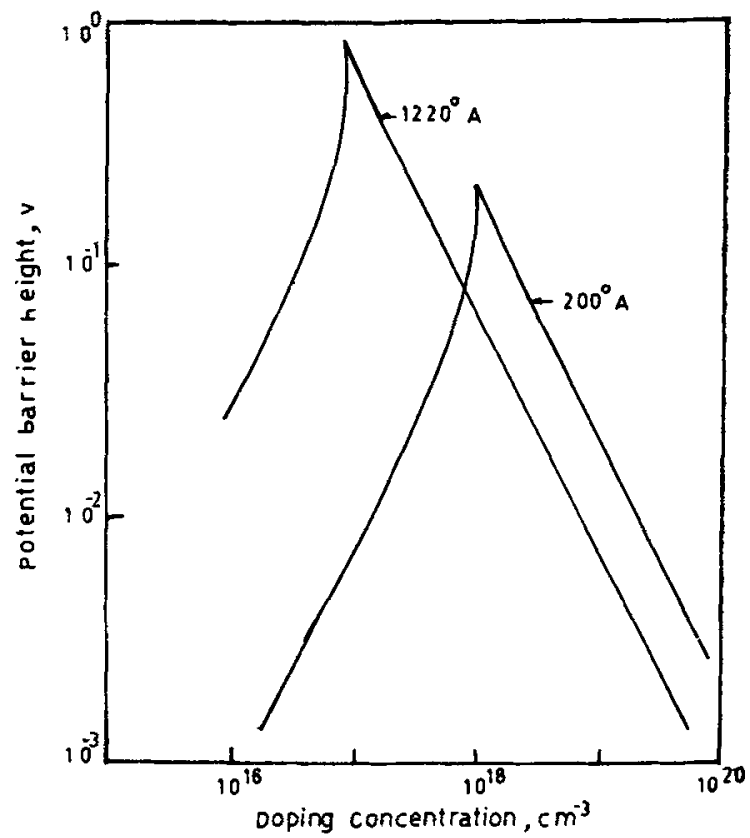

Figure 5. Theoretical variation of potential barrier height with doping concentration for different grain sizes.

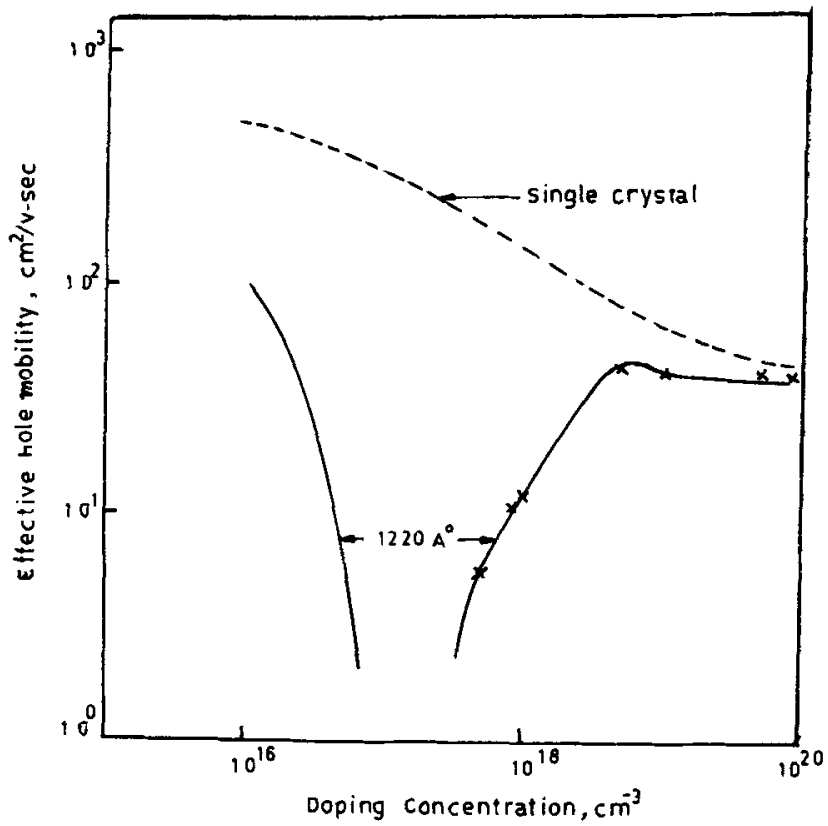

Figure 6. Theoretical variation of the effective mobility with doping concentration at room temperature for grain size $d=1220 \AA$ ( +, Lu et al $1981 \mathrm{~b}$; --, present work).

Figure 6 shows the variation of the effective mobility in polysilicon as a function of doping concentration for $d=1220 \AA$. The values of single crystal are assumed for the parameters $m_{n}^{*}, n_{i}, E_{g}, \varepsilon$ and $\mu$. There is a good agreement between our 
computed results and the available experimental data (Lu et al 1981b) As doping concentration increases, $\mu^{*}$ decreases and reaches a minimum at $N=N^{*}$. This is because $V_{g}$ is maximum at $N=N^{*}$. After this doping concentration, it approaches the corresponding monocrystalline mobility. At high doping levels, $\mu^{*}$ is mainly governed by crystallite mobility.

Figure 7 shows the variation of polysilicon resistivity as a function of doping concentration for various grain sizes. It is quite evident that our calculated results are in good agreement with available experimental data (Seto 1975; Lu et al 1981b). At low doping concentrations (i.e. $N<N^{*}$ ), the resistivity of polysilicon is much higher than that of mono-crystalline silicon because, in this range, the average carrier concentration $P^{*}$ is very small. Here resistivity is mainly governed by $P^{*}$. At intermediate doping concentrations, even a very small increase in doping concentration causes an abrupt and large drop in resistivity. This sharp drop in resistivity is due to decrease in $V_{g}$ and consequently the increases in $P^{*}$ and $\mu^{*}$. At high doping levels (i.e. $N \gg N^{*}$ ), the effect of $\mu^{*}$ and $P^{*}$ is very small and $\rho$ approaches the monocrystalline resistivity. From the figure it is also clear that for the intermediate doping concentrations the polysilicon resistivity drops sharply as the grain size increases from $200 \AA$ to $1220 \AA$. Figure 8 shows the variation of polysilicon resistivity with doping concentration at different temperatures. It is clear that at high doping concentrations, resistivity is independent of temperature. Thus, the resistivity of polysilicon is very sensitive to the doping concentration and for small grain sizes.

A critical glance at the graphs shown in figure 8 reveals that there is good agreement between available experimental data and our computed results using the proposed simple model in which only thermionic emission effect has been accounted for. Although the model used by Lu et al (1983) considered all the three

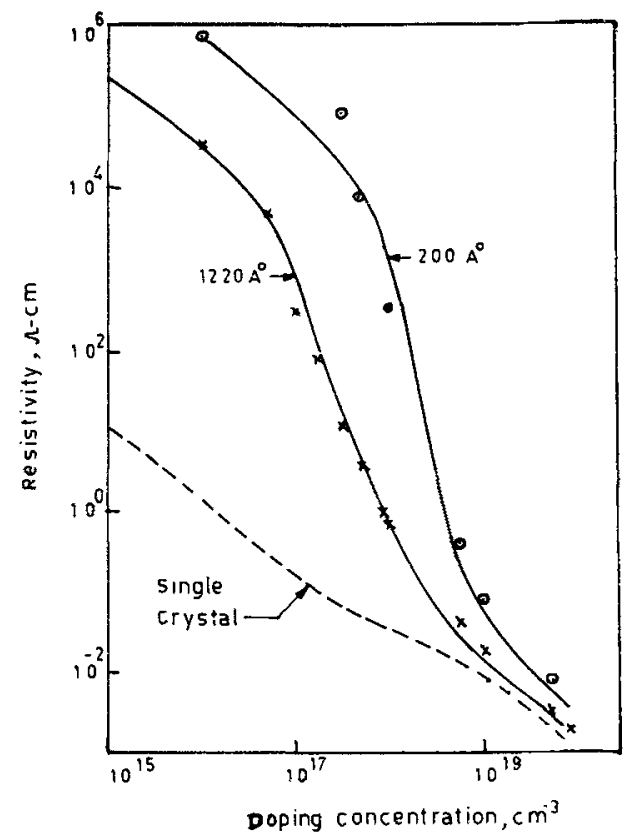

Figure 7. Theoretical variation of polysilicon resistivity with doping concentration at room temperature for different grain sizes $(\odot$, Seto 1975 ; + , Lu et al; -, present work.). 


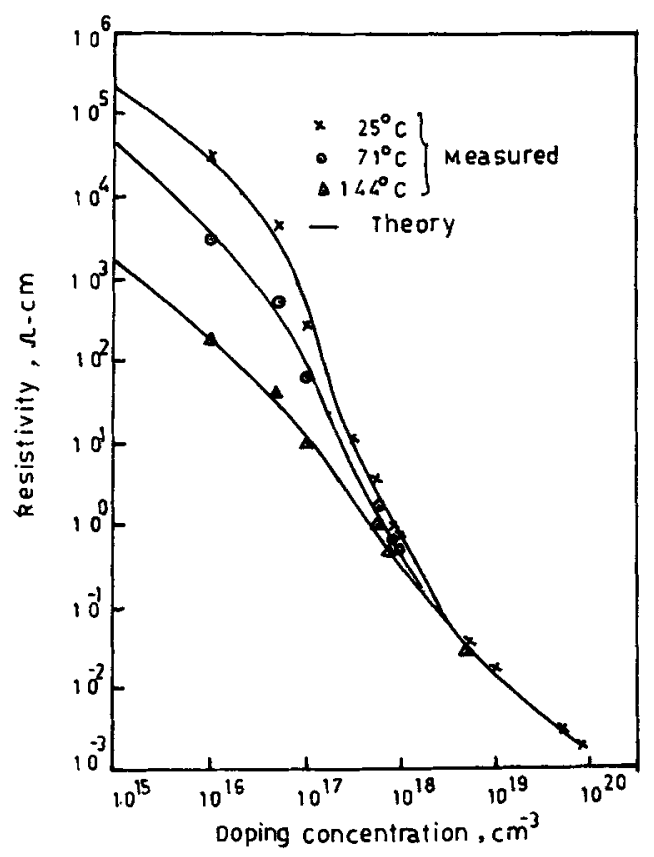

Figure 8. Theoretical variation of polysilicon resistivity with doping concentration at different temperatures for grain size $d=1220 \AA+\odot, \triangle$ data are from Lu et al (1981b).

effects viz. thermionic field emission scattering, thermionic field emission and thermionic emission, yet the reported agreement between their experimental data and theoretical results is not as good as that obtained by using the simple model proposed on the consideration of thermionic emission only.

\section{Acknowledgement}

One of the authors (SS) is grateful to $\operatorname{Dr} R$ Pranaitis, Department of Semiconductor Physics, Vilnius State University, USSR for some useful discussions. Some of the theoretical details were worked out during the stay of the author as visiting scientist at Vilnius.

\section{References}

Abo-Namous S A 1987 Physs. Status Solidi A102 703

Choudhary P R and Hover P L 1983 J. Electrochem. Soc. 1201761

Cowher M E and Sedwich T O 1972 J. Electrochem. Soc. 1191565

Fripp A L and Slack L H 1973 J. Electrochem. Soc. 120145

Henry C H, Logan R A and Merrit F R 1978 J. Appl. Phys. 493530

Kamins T I 1971 J. Appl. Phys. 424357

Lu N C C, Gerzberg L and Meindl J D 1980 IEEE Trans, Electron Device Lett. 138

Lu N C C, Gerzberg L, Lu C Y and Meindl J D 1981a IEEE Trans. Electron Device Lett. 295

Lu N C C, Gerzberg L, Lu C Y and Meindl J D 1981b IEEE Trans. Electron Devices 28818

Lu N C C, Gerzberg L, Lu C Y and Meindl J D 1983 IEEE Trans. Electron Devices 30137 
Mandurah M M, Saraswat K C and Kamins T I 1981 IEEE Trans. Electron Devices 28 1163, 1171 Muller R S and Kamins T I 1977 in Devices electronics for integrated circuits (New York: Wiley) Chap. 1 Murota J and Sawai T 1982 J. Appl. Phys. 533702

Pantalides S T, Selloni A and Car R 1985 Solid State Electron. 2817

Samaj L 1987 Phys. Status Solidi A100157

Seto J Y W 1975 J. Appl. Phys. 465247

Sze S M 1981 in Physics of semiconductor devices 2nd Ed (New York: Wiley) Chap. 1, 4, 8

Upreti N K and Singh S 1987 Proc. Solid State Phys. Symp. BARC, Bombay p. 423

Wolf H F 1969 in Silicon semiconductor data (Oxford: Pergamon) Ch. 2 